\title{
Development of a particle swarm optimisation model for estimating the homogeneity of a mixture inside a newly designed CNG-H2-AIR mixer for a dual fuel engine: An experimental and theoretic study
}

\begin{abstract}
Many research works have intended to enhance fuel economy and decrease emissions during conversion from a diesel engine to a dual fuel engine. However, the majority of these works do not take into account enhancement of homogeneity of the mixture inside the engine and precise control of the air fuel ratio. This deficiency can cause higher emissions, greater brakespecific fuel consumption, and likely knocking. Conversely, there is limited research pertaining to empirical equations for projecting the mixture's homogeneity. In this study, a new air-fuel mixer was devised, produced and tested. For the air-gaseous fuel mixer, the proposed design was meant to be appropriate for mixing air with hydrogen and CNG. It was also designed in such a way that it would result into extremely homogeneous mixing for the gaseous fuel as it mixes with air and exhibits high uniformity index (UI). Lastly, it is also meant to promote easy connection with an electronic control unit so that the air-gaseous fuel ratio could be accurately controlled for varying engine speeds. To optimise the homogeneity within the new mixer, fifteen varying mixer models having 116 cases were made in order to study how the location, diameter, and number of holes within the mixer affect the mixture's homogeneity and distribution under $\mathrm{ACNGR}=34.15$ and $\mathrm{AHR}=74.76$. Afterwards, the distribution, flow behaviour, and homogeneity of the mixture within the new mixer models were checked using computational fluid dynamics analysis software. Based on the simulation results, it was discovered that the best uniformity index (UI) values were achieved for models $7 /$ case 48 . Based on the simulation results, a fairly simple method was then developed to estimate the mixture's homogeneity (UI) from the new models of the mixer. The basis of the proposal model (empirical equation) is from the best values determined for the unknown constant $\mathrm{F}$ so that the equation for UI estimation could be formulated. The particle swarm optimisation (PSO) algorithm was used to solve an optimisation problem and achieve this outcome. The outcomes indicated that the built model could precisely project the UI values.
\end{abstract}

Keyword: Homogeneity of the mixture; AIR-CNG-hydrogen mixer; Internal combustion engines; Computational fluid dynamics; Particle swarm optimisation 